\title{
Magnetic Phase Transition of MnBi under High Magnetic Fields and High Temperature
}

\author{
Keiichi Koyama ${ }^{1, *}$, Tetsuya Onogi ${ }^{1}$, Yoshifuru Mitsui ${ }^{1}$, Yuko Nakamori ${ }^{2}$, \\ Shin-ichi Orimo ${ }^{2}$ and Kazuo Watanabe ${ }^{1}$ \\ ${ }^{1}$ High Field Laboratory for Superconducting Materials, Institute for Materials Research, \\ Tohoku University, Sendai 980-8577, Japan \\ ${ }^{2}$ Institute for Materials Research, Tohoku University, Sendai 980-8577, Japan
}

\begin{abstract}
Magnetization measurements and differential thermal analysis (DTA) of polycrystalline MnBi were carried out in magnetic fields up to $14 \mathrm{~T}$ and in 300-773 K, in order to investigate the magnetic phase transition. The magnetic phase transition temperature $\left(T_{\mathrm{t}}\right)$ at a zero magnetic field is $628 \mathrm{~K}$ and linearly increases with increasing fields up to $14 \mathrm{~T}$ at the rate of $2 \mathrm{KT}^{-1}$. A metamagnetic transition between the paramagnetic and field-induced ferromagnetic states was observed just above $T_{\mathrm{t}}$. The exothermic and endothermic peaks were detected in the magnetic field dependence of DTA signals in 626-623 K, which relates to the metamagnetic transition. The obtained results were discussed on the basis of a mean field theory. [doi:10.2320/matertrans.MAW200721]
\end{abstract}

(Received April 23, 2007; Accepted July 6, 2007; Published August 22, 2007)

Keywords: MnBi compound, high magnetic field, differential thermal analysis, metamagnetic transition, first order transition

\section{Introduction}

Binary compound $\mathrm{MnBi}$ with a NiAs-type hexagonal structure (low temperature phase: LTP) exhibits unique magnetic and structural properties. ${ }^{1,2)}$ The compound has a uniaxial magnetic anisotropy along the $c$-axis at room temperature (RT). With decreasing temperature from RT, the magnetic moment lies in the $c$-plane at $84 \mathrm{~K}^{3)}$ Heikes $^{4)}$ and Roberts ${ }^{5}$ ) reported that the magnetic moment $m$ of LTP extrapolates to a value of $3.9 \mu_{\mathrm{B}} / \mathrm{Mn}$ at $0 \mathrm{~K}$. The mean field calculations on $m$ of LTP-MnBi indicated that the Curie temperature $T_{\mathrm{C}}$ would reach up to $720 \mathrm{~K}$. $^{6}$

However, with increasing temperature from RT, LTP$\mathrm{MnBi}$ shows a first-order magnetic phase transition from the ferromagnetic (FM) to paramagnetic $(\mathrm{PM})$ state at $T_{\mathrm{t}} \sim$ $660 \mathrm{~K}$, accompanied by a structural transformation from the NiAs-type to distorted $\mathrm{Ni}_{2}$ In-type hexagonal structure (high temperature phase: HTP). ${ }^{5}$ The cell volume slightly shrinks by $0.03 \%$ in the transformation from LTP to HTP. After that, Chen reported that HTP-MnBi was a separate compound with chemical formula $\mathrm{Mn}_{1.08} \mathrm{Bi}^{7}{ }^{7)}$ According to the $\mathrm{Mn}$-Bi phase diagram, ${ }^{7)}$ the phase transition of $\mathrm{MnBi}$ at $T_{\mathrm{t}}$ upon heating is associated with a peritectic decomposition of $\mathrm{MnBi}$ (LTP) into $\mathrm{Mn}_{1.08} \mathrm{Bi}$ (HTP) and liquid Bi. Recently, from magnetic measurements for $\mathrm{MnBi}$, Liu et al. reported that $T_{\mathrm{t}}$ increases linearly with increasing magnetic fields up to $10 \mathrm{~T}$ at the rate of $\sim 2 \mathrm{KT}^{-1}{ }^{8)}$ These results suggest that the first-order phase transition with the peritectic composition and the decomposition between $\mathrm{MnBi}$ and $\mathrm{Mn}_{1.08} \mathrm{Bi}$ can be controlled by magnetic fields.

In this study, in order to investigate the magnetic phase transition of LTP-MnBi under high magnetic fields and high temperature, we have carried out magnetization measurements and differential thermal analysis (DTA) in the temperature $T$ range from 300 to $773 \mathrm{~K}$ under magnetic fields $B$ up to $14 \mathrm{~T}$.

*Corresponding author, E-mail: kkoyama@imr.tohoku.ac.jp

\section{Experimental}

Polycrystalline MnBi was prepared by arc-melting the mixture of stoichiometric amounts of pure elements (Mn, 3N; $\mathrm{Bi}, 5 \mathrm{~N})$ in an argon atmosphere. The button ingot was turned over, re-melted several times. After that, the ingot was annealed at $573 \mathrm{~K}$ for 5 hours in a quartz tube with an argon atmosphere and then quenched in water. X-ray powder diffraction measurements were carried out with $\mathrm{Cu} \mathrm{K} \alpha$ radiation at RT. The sample was confirmed to be LTP with the NiAs-type crystal structure with a small amount of Bi, but we did not observed the reflection peaks of HTP. The lattice parameters $a$ and $c$ were determined to be $0.4310 \mathrm{~nm}$ and $0.6076 \mathrm{~nm}$ at RT, respectively, which are comparable to the reported data of the LTP phase. ${ }^{4,5,7-12)}$

Using a Farady-force magnetometer, magnetization $M$ was measured in the temperature range from 300 to $620 \mathrm{~K}$ and in magnetic fields up to $10 \mathrm{~T}$. The experimental setup of the magnetization measurements was described in Ref. 13) in detail.

DTA was carried out under an argon atmosphere (ambient pressure) in the temperature $T$ range from 300 to $773 \mathrm{~K}$ and in magnetic fields $B$ up to $14 \mathrm{~T}$. In this measurement, $\mathrm{Al}_{2} \mathrm{O}_{3}$ is utilized as a reference sample. A detailed description of the high-field DTA technique can be found in Ref. 14).

\section{Results}

Figure 1 shows the DTA curves of $\mathrm{MnBi}$ in the various magnetic fields up to $14 \mathrm{~T}$. The data were taken in heating process with the rate of $3 \mathrm{Kmin}^{-1}$. In a zero magnetic field, three endothermic peaks were observed: the melting of $\mathrm{Bi}$ at $535 \mathrm{~K}\left(T_{\mathrm{m}}{ }^{\mathrm{Bi}}\right)$, the phase transition from LTP to HTP at $638 \mathrm{~K}$ $\left(T_{\mathrm{t}}\right)$, and the peritectic temperature $\left(T_{\mathrm{m}}{ }^{\mathrm{MnBi}}\right)$ of $\mathrm{HTP}$ at $719 \mathrm{~K}$. Here, these transition temperatures were determined at the onset of the endothermic peaks. As seen in this figure, $T_{\mathrm{m}}{ }^{\mathrm{Bi}}$ and $T_{\mathrm{m}}{ }^{\mathrm{MnBi}}$ are not affected by applying $B$, but $T_{\mathrm{t}}$ increases with increasing $B$. 


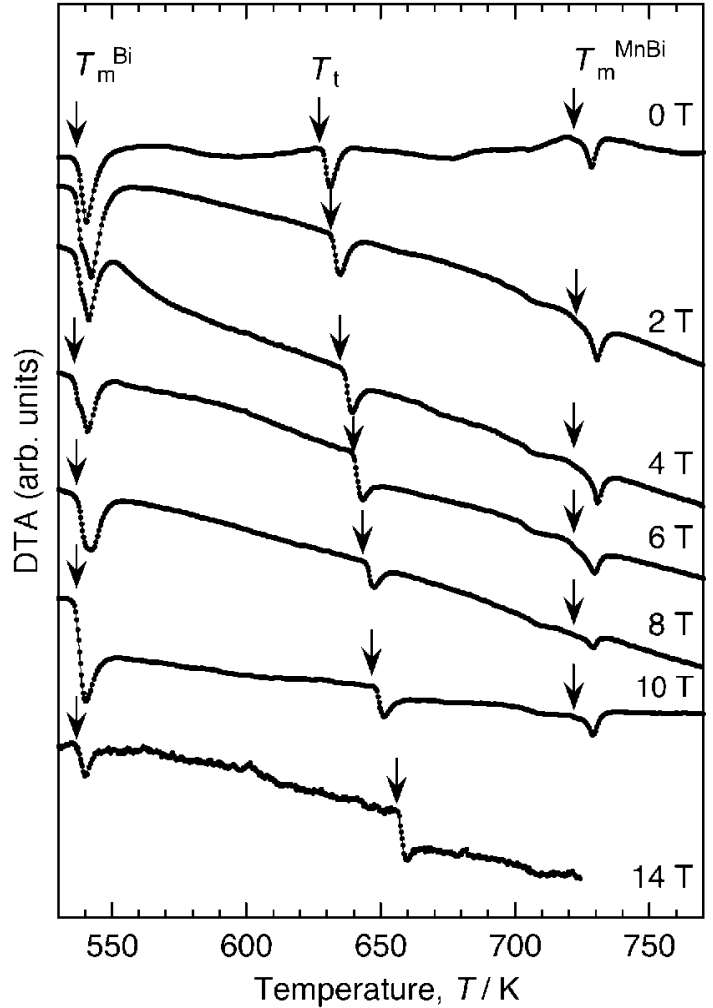

Fig. 1 DTA curves of MnBi at the various magnetic fields up to $14 \mathrm{~T}$. The data were taken in heating process. The vertical arrows indicate the determined phase transition temperatures.

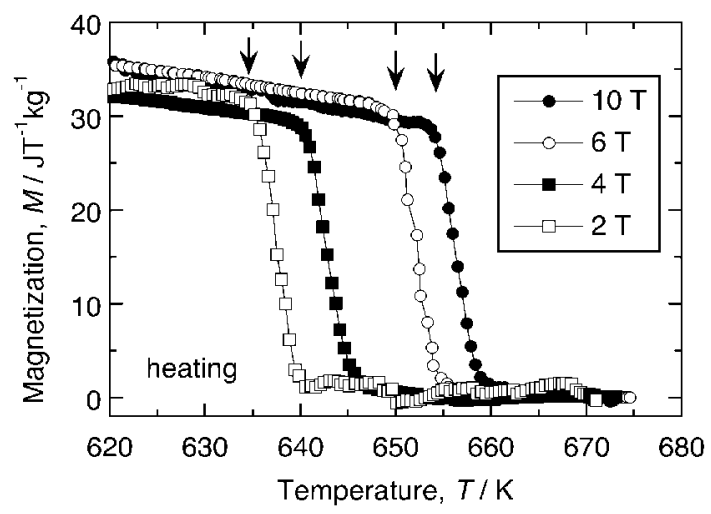

Fig. 2 Temperature dependence of the magnetization of $\mathrm{MnBi}$ in various magnetic fields up to $10 \mathrm{~T}$ at the vicinity of the magnetic phase transition temperature, $T_{\mathrm{t}}$. The vertical arrows indicate the determined $T_{\mathrm{t}}$.

Figure 2 shows the typical results of the temperature dependence of the magnetization ( $M-T$ curves) of $\mathrm{MnBi}$ in various magnetic fields up to $10 \mathrm{~T}$. Here, the data were taken in heating process. In this figure, the vertical arrows indicate the determined magnetic phase transition temperatures. With increasing $T, M$ abruptly vanishes at $T_{\mathrm{t}}$ even in higher magnetic field, which indicates that the magnetic transition is a first-order. Liu et al. reported that the thermal hysteresis of $T_{\mathrm{t}}$ is about $10 \mathrm{~K}^{8)}$

Figure 3 shows the magnetic field dependence of $T_{\mathrm{t}}$ of MnBi. With increasing $B$ up to $14 \mathrm{~T}, T_{\mathrm{t}}$ determined by the DTA measurements increases linearly by $2 \mathrm{KT}^{-1}$, though the

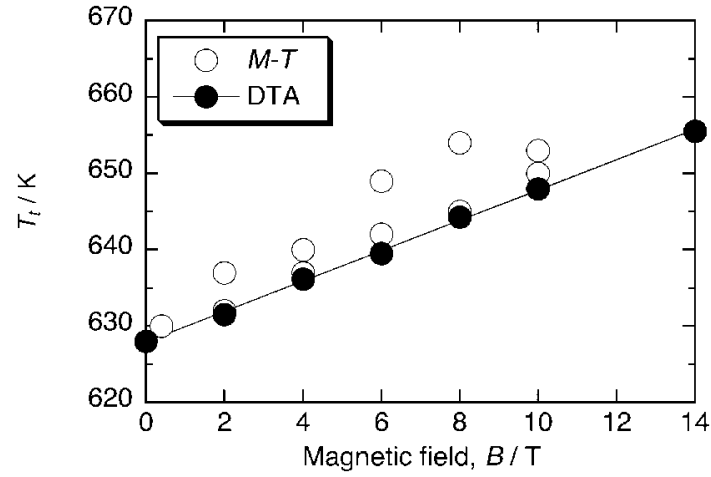

Fig. 3 Magnetic field dependence of the magnetic phase transition temperature $T_{\mathrm{t}}$ of $\mathrm{MnBi}$. The solid and open circles indicate $T_{\mathrm{t}}$ determined by DTA and the magnetic data ${ }^{13)}$ respectively. The solid line indicates the result determined by least squares calculation for $T_{\mathrm{t}}$ estimated by DTA.

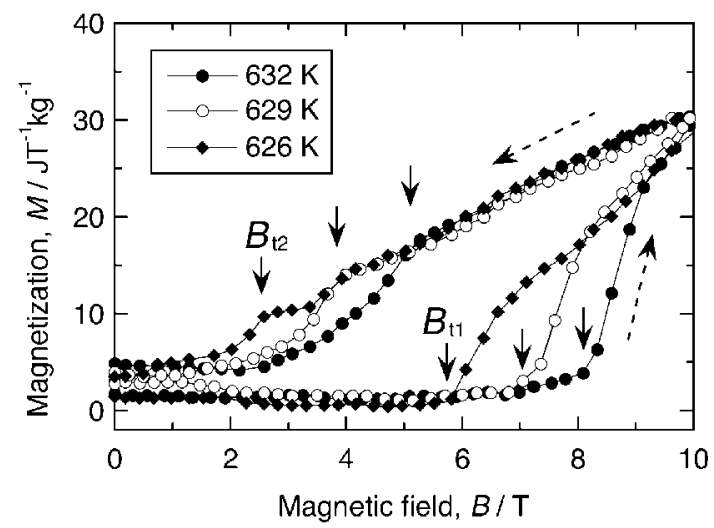

Fig. 4 Magnetization curves of $\mathrm{MnBi}$ at 626, 629 and $632 \mathrm{~K}$. Here, all curves were measured after zero-field cooling from $650 \mathrm{~K}$ (paramagnetic phase). The vertical arrows indicate the determined metamagnetic transition fields from the paramagnetic to field-induced ferromagnetic state $\left(B_{\mathrm{t} 1}\right)$ and from the field-induced ferromagnetic to paramagnetic state $\left(B_{\mathrm{t} 2}\right)$. The broken arrows indicate the magnetization processes.

data $^{13)}$ determined by the $M-T$ curves were somewhat scattered. The obtained results show that the endothermic at $T_{\mathrm{t}}$ in Fig. 1 is due to the first-order magnetic phase transition from the FM to PM state. By applying $B$, the only single endothermic peak at $T_{\mathrm{t}}$ shifts to higher temperature, and no extra peak was observed. This suggests that the LTPHTP structural transformation with the peritectic decomposition is also affected by the magnetic field, accompanied by the first-order magnetic phase transition.

Figure 4 shows the typical results of the magnetization curves ( $M-B$ curves) of $\mathrm{MnBi}$ at 626,629 and $632 \mathrm{~K}$. Here, all curves were measured after zero-field cooling from $650 \mathrm{~K}$ (PM phase). This figure clearly shows the metamagnetic transition between the PM and field-induced ferromagnetic (FFM) state in MnBi. The vertical arrows in Fig. 4 indicate the determined metamagnetic transition fields from the PM to FFM state $\left(B_{\mathrm{t} 1}\right)$ and from the FFM to PM state $\left(B_{\mathrm{t} 2}\right)$. With increasing $T, B_{\mathrm{t} 1}$ and $B_{\mathrm{t} 2}$ shift to higher magnetic field, and the magnetic hysteresis is approximately $3.3 \mathrm{~T}$.

Figure 5 shows the typical results of the magnetic field dependence of the DTA signal for MnBi at 626, 628, 630 and $632 \mathrm{~K}$. Here, all field dependent curves were measured after 


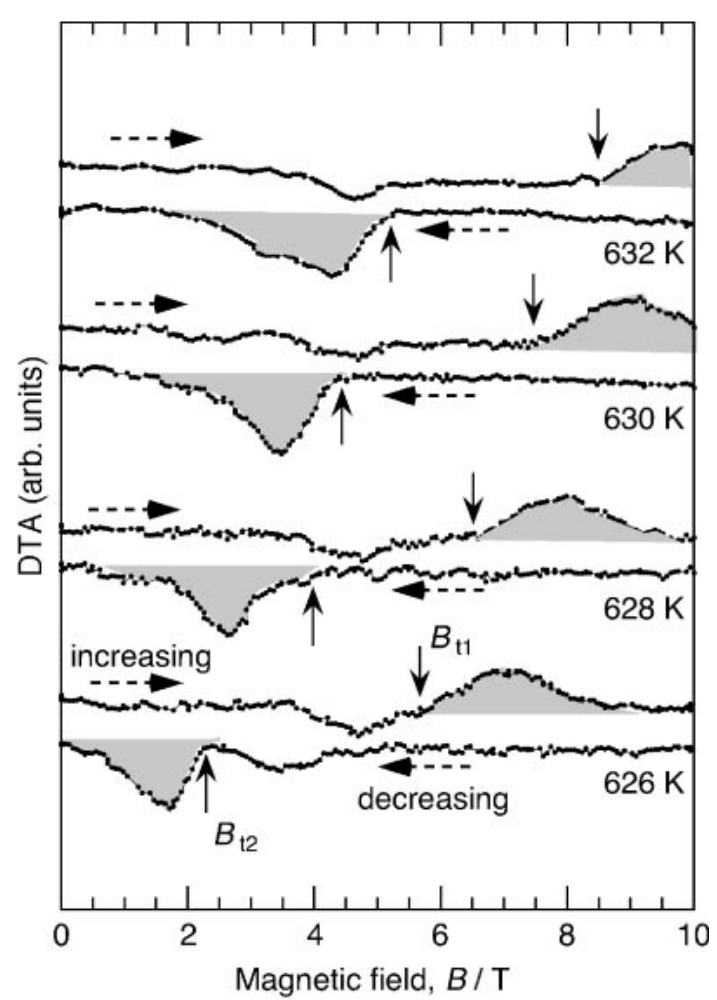

Fig. 5 Magnetic field dependence of the DTA signal for MnBi at 626, 628, 630 and $632 \mathrm{~K}$. Here, all field-dependent curves were measured after zerofield cooling from $650 \mathrm{~K}$ (paramagnetic phase). The vertical arrows indicate the determined phase transition fields $B_{\mathrm{t} 1}$ and $B_{\mathrm{t} 2}$ for increasing and decreasing magnetic fields, respectively.

zero-field cooling from $650 \mathrm{~K}$ (PM phase). At $626 \mathrm{~K}$, we observed the exothermic and endothermic peaks at $5.7 \mathrm{~T}$ $\left(=B_{\mathrm{t} 1}\right)$ for increasing $B$ and at $2.5 \mathrm{~T}\left(=B_{\mathrm{t} 2}\right)$ for decreasing $B$, respectively. The arrows in Fig. 5 indicate $B_{\mathrm{t} 1}$ and $B_{\mathrm{t} 2}$ that were determined at the onset of the DTA peaks. With increasing $T, B_{\mathrm{t} 1}$ and $B_{\mathrm{t} 2}$ shift to higher magnetic field. Considering the $M-B$ curves (Fig. 4), the exothermic and endothermic peaks in Fig. 5 are due to the metamagnetic transition from the PM to FFM state and from the FFM to PM state, respectively. That is, Fig. 5 shows the magnetocaloric effect accompanied by the metamagnetic transition. In this study, a small dip was also observed at the vicinity of $4.7 \mathrm{~T}$ for increasing $B$, which is independent of temperature variation. At present, the origin of the dip is unclear. However, the dip does not relate essentially to the magnetic properties of $\mathrm{MnBi}$, because it is inconsistent with the $M-B$ curves.

According to the Mn-Bi phase diagram ${ }^{7)}$ and the structural properties $^{5)}$ of $\mathrm{MnBi}$, the compound exhibits the phase transition between FM-MnBi (NiAs-type) and $\mathrm{PM}-\mathrm{Mn}_{1.08} \mathrm{Bi}$ (distorted $\mathrm{Ni}_{2} \mathrm{In}$-type). That is, the metamagnetic transition probably occurs, accompanied by the structural and chemical formula changes. Therefore, the observed $M-B$ curves are out of shape at the metamagnetic transition (Fig. 4). In Fig. 5, the small dip of the DTA signal is seen at the vicinity of $3.5 \mathrm{~T}$ at $626 \mathrm{~K}$ for decreasing $B$. The origin of the dip is also unclear at present. However, since the two-step like $M-B$ curve is observed at $626 \mathrm{~K}$, the small dip at $\sim 3.5 \mathrm{~T}$ may be due to the magnetic transition.

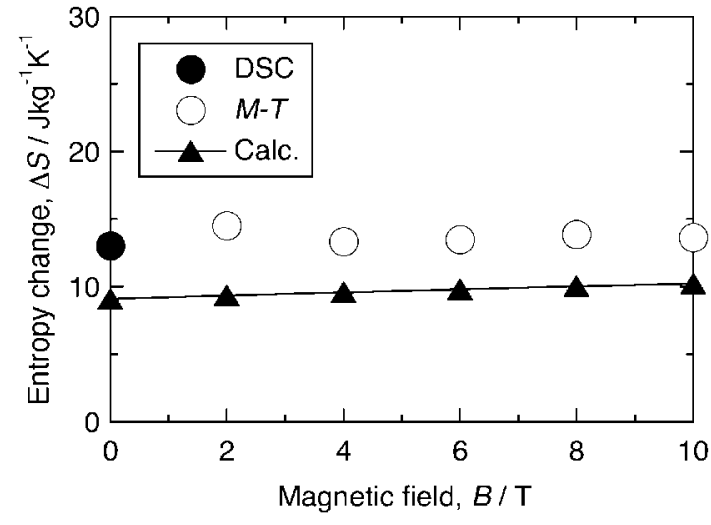

Fig. 6 Magnetic field dependence of the entropy change of $\mathrm{MnBi}$ at the vicinity of the magnetic phase transition temperature. The solid circles indicate the data determined by differential scanning calorimeter (DSC) analysis under a zero magnetic field. The open circles are the data estimated by Clausius-Clapayron equation from the $M-T$ curves. ${ }^{13)}$ The solid triangles indicate the magnetic entropy change calculated by a mean field model.

\section{Discussion}

In this section, we will discuss the entropy change at the vicinity of $T_{\mathrm{t}}$ and the magnetic properties of LTP-MnBi. Using Clausius-Clapayron equation, the entropy change $\Delta S$ between the FM(FFM) and PM phase at $T_{\mathrm{t}}$ can be estimated from the $M-T$ data. ${ }^{13)}$ In Fig. 6, the magnetic field dependence of $\Delta S$ is shown by the open circles. As seen in this figure, $\Delta S$ is $\sim 14 \mathrm{Jkg}^{-1} \mathrm{~K}^{-1}$ at $T_{\mathrm{t}}$, which is almost constant in $0 \leq B \leq 10 \mathrm{~T}$. This value is good agreement with our result $\left(13 \mathrm{Jkg}^{-1} \mathrm{~K}^{-1}\right)$ deduced from DSC analysis at a zero field, as shown by the solid circle in Fig. 6. This large entropy change is comparable with that of $\mathrm{Gd}_{5} \mathrm{Ge}_{2} \mathrm{Si}_{2}{ }^{15)}$ or $\mathrm{MnGeP}_{0.45} \mathrm{As}_{0.55}{ }^{16)}$ that have attracted interest as magnetic refrigeration materials. In these materials, it is supposed that the large entropy change originates from the disappearance of the large magnetic moment, accompanied by the structural transformation.

In order to estimate the contribution of the magnetic entropy change $\Delta S_{\mathrm{m}}$ to $\Delta S$ in $\mathrm{MnBi}$ at $T_{\mathrm{t}}$, we calculated the magnetic free energy on the basis of a simple mean field model. Here, we assume that the orbital angular momentum is quenched, and the magnetic moment $m$ of $\mathrm{MnBi}$ originates from the thermal average of the total spin angular momentum $S$ of $\mathrm{Mn}$. In this model, the magnetization $M(T, B)$ at temperature $T$ and applied magnetic field $B$ can be expressed as follows:

$$
\begin{aligned}
M(T, B) & =N m \\
& =2 N S \mu_{\mathrm{B}} B_{\mathrm{J}}(\alpha),
\end{aligned}
$$

where

$$
\alpha=\frac{2 S \mu_{\mathrm{B}}(\lambda M+B)}{k_{\mathrm{B}} T}
$$

and $B_{\mathrm{J}}(\alpha)$ is the Brillouin function, $N$ the number of magnetic atoms per unit volume, $S$ the total angular spin momentum, $k_{\mathrm{B}}$ the Boltzmann constant, $\mu_{\mathrm{B}}$ the Bohr magneton, $\lambda$ the mean field constant. The magnetic free energy per molecule of the system is given by 


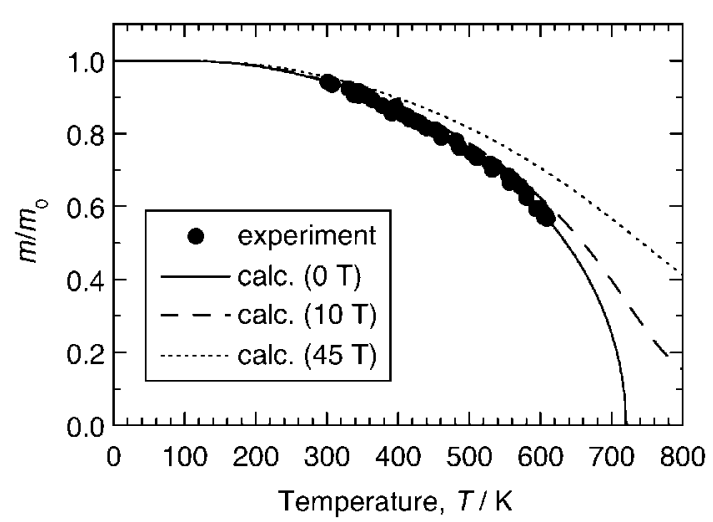

Fig. 7 Temperature dependence of the experimental (solid circles) and calculated magnetic moments $m$ curves for $B=0,10$ and $45 \mathrm{~T}$. The moment is normalized by the spontaneous magnetic moment $m_{0}$.

$$
F=-k_{\mathrm{B}} T \ln Z-m(\lambda M+B) .
$$

where, $Z$ is the partition function defined as

$$
Z=\operatorname{Tr} \exp \left[-2 \mu_{\mathrm{B}} S(\lambda M+B) / k_{\mathrm{B}} T\right] .
$$

Using these equations, we calculated $F$ and the magnetic entropy $S_{\mathrm{m}}(=\Delta F / \Delta T)$. The neutron diffraction measurements by Roberts showed $m=3.9 \mu_{\mathrm{B}} / \mathrm{Mn}$ in LTP-MnBi at $77.3 \mathrm{~K}^{5)}$ Therefore, this calculation was carried out for $S=2$ $\left(\mathrm{Mn}^{3+}\right)$, and the spontaneous magnetic moment $\left(m_{0}\right)$ is $4 \mu_{\mathrm{B}} /$ $\mathrm{Mn}$ at $0 \mathrm{~K}$.

Figure 7 shows the temperature dependence of the experimental and calculated magnetic moments $m$ of LTPMnBi for $B=0,10$ and $45 \mathrm{~T}$. Here, the value is normalized by $m_{0}$. In this calculation, we used the maximum parameter of $B=45 \mathrm{~T}$, because this field is utilized as a steady magnetic field. ${ }^{17)}$ Using the parameter $\lambda=6.33 \mathrm{kgT}^{2} \mathrm{~J}^{-1}$ $\left(T_{\mathrm{C}}=720 \mathrm{~K}\right)$ and $B=0 \mathrm{~T}$, the calculation represents the experimental $M-T$ data well, which is consistent with previous report. $\left.{ }^{6}\right)$

Here, assuming that the calculated $m$ of LTP-MnBi vanishes at $T_{\mathrm{t}}$ determined from the experimental data (Fig. 2), we calculated $\Delta S_{\mathrm{m}}\left(=S_{\mathrm{m}}(m)-S_{\mathrm{m}}(m=0)\right)$ in the various magnetic fields up to $10 \mathrm{~T}$ using the same parameter $\lambda$. The magnetic field dependence of $\Delta S_{\mathrm{m}}$ is shown by the triangles in Fig. 6. At $B=0 \mathrm{~T}, \Delta S_{\mathrm{m}}$ is $9.1 \mathrm{Jkg}^{-1} \mathrm{~K}^{-1}$, which is almost constant in $0 \mathrm{~T} \leq B \leq 10 \mathrm{~T}$. In this field range, calculated $\Delta S_{\mathrm{m}}$ is approximately $80 \%$ of the experimental value. This result suggests that the entropy change is mainly due to the magnetic entropy change that originates from the disappearance of the large magnetic moment in $\mathrm{MnBi}$ at $T_{\mathrm{t}}$, and the magnetic free energy plays an important role in the first-order phase transition. However, strictly speaking, the calculated $\Delta S_{\mathrm{m}}$ slightly increases with increasing $B$, which is due to the increase of the filed-induced magnetic moment in LTP-MnBi. It is required to precisely measure the magnetic moment under high magnetic fields at the vicinity of $T_{\mathrm{t}}$.

As mentioned above, we can see that only one endothermic peak at $T_{\mathrm{t}}$ on the DTA data (Fig. 1) shifts to higher temperature with increasing $B$, indicating that the magnetic field affects the first-order magnetic and structural transition between FM-MnBi with the NiAs-type structure (LTP) and PM- $\mathrm{Mn}_{1.08} \mathrm{Bi}$ with the distorted $\mathrm{Ni}_{2}$ In-type structure (HTP).
The transition at $T_{\mathrm{t}}$ probably occurs to minimize the magnetic and elastic energies between FM-LTP MnBi and PM-HTP $\mathrm{Mn}_{1.08} \mathrm{Bi}$. Applying a magnetic field to the compound, the decrease in magnetic free energy of FM (FFM)-LTP MnBi is much larger than that of PM-HTP $\mathrm{Mn}_{1.08} \mathrm{Bi}$ because of the addition of the Zeeman energy. Therefore, we observe the increase of $T_{\mathrm{t}}$ by applying $B$ and the metamagnetic transition in this compound.

The reports in Refs. 8) and 13) and our DTA result show that $T_{\mathrm{t}}$ linearly increases at the rate of $2-2.3 \mathrm{KT}^{-1}$ in fields up to $14 \mathrm{~T}$. Assuming that this rate is constant for further high temperature and high magnetic field, $T_{\mathrm{t}}$ will reach up to the peritectic temperature $\left(T_{\mathrm{m}} \mathrm{MnBi} \sim 720 \mathrm{~K}\right)$ of HTP by applying $B \sim 45$ T. As shown in Fig. 7, LTP-MnBi may have a fieldinduced magnetic moment in $B=45 \mathrm{~T}$ at $720 \mathrm{~K}$. This suggests that we can control the first-order magnetic and structural transition of $\mathrm{MnBi}$ till the peritectic temperature $T_{\mathrm{m}}{ }^{\mathrm{MnBi}}$ and solidify $\mathrm{MnBi}$ without the PM phase by high magnetic fields. Therefore, from the point of view of magneto-science, it is interested in controlling the magnetic, structural and chemical formula and in synthesizing magnetic material MnBi under high magnetic fields.

\section{Summary}

Magnetization measurements and thermal analysis of MnBi were performed in magnetic fields up to $14 \mathrm{~T}$ and in $300-773 \mathrm{~K}$. The magnetic phase transition temperature $\left(T_{\mathrm{t}}\right)$ at a zero magnetic field is $628 \mathrm{~K}$, which linearly increases by $2 \mathrm{KT}^{-1}$ with increasing magnetic fields up to $14 \mathrm{~T}$. Furthermore, the exothermic and endothermic peaks were observed on the magnetic field dependence of DTA signals at 626$623 \mathrm{~K}$, which relates to the metamagnetic transition between the paramagnetic and field-induced ferromagnetic states. The entropy change at $T_{\mathrm{t}}$ was estimated to be $14 \mathrm{Jkg}^{-1} \mathrm{~K}^{-1}$ for $2 \mathrm{~T}$ from the magnetic data using Clausius-Clapayron equation, which is almost constant for magnetic fields up to $10 \mathrm{~T}$. The simple mean field model represents the temperature dependence of the magnetization of $\mathrm{MnBi}$. The calculated magnetic entropy change is $9.1 \mathrm{Jkg}^{-1} \mathrm{~K}^{-1}$ at $T_{\mathrm{t}}$ in a zero field, which is approximately $80 \%$ of the experimental data. The obtained results suggest that the entropy change is mainly due to the magnetic entropy change at $T_{\mathrm{t}}$.

\section{Acknowledgments}

The work was carried out at the High Field Laboratory for Superconducting Materials, Institute for Materials Research, Tohoku University. The authors are very much indebted to Dr. H. Yoshida for valuable discussions regarding to the sample preparation. This work was supported by a Grant inAid for Scientific Research from the Ministry of Education, Science and Technology, Japan.

\section{REFERENCES}

1) C. Gullaud: J. Phys. Radium 12 (1951) 143-143.

2) C. Gullaud: J. Phys. Radium 12 (1951) 223-227.

3) C. Gullaud: J. Phys. Radium 12 (1951) 492-497.

4) R. R. Heikes: Phys. Rev. 99 (1955) 446-447. 
5) B. W. Roberts: Phys. Rev. 104 (1956) 607-616.

6) B. A. Huberman and W. Streifer: Phys. Rev. B 12 (1975) 2741-2746.

7) T. Chen: J. Appl. Phys. 45 (1974) 2358-2360.

8) Y. Liu, J. Zhang, S. Cao, G. Jia, X. Zhang, Z. Ren, X. Li, C. Jing and K. Deng: Solid State Commun. 138 (2006) 104-109.

9) H. Yoshida, T. Shima, T. Takahashi and H. Fujimori: Mater. Trans. 40 (1999) 455-458.

10) H. Yoshida, T. Shima, T. Takahashi, H. Fujimori, S. Abe, T. Kaneko, T. Kanomata, T. Suzuki: J. Alloy. Comp. 317-318 (2001) 297-301.

11) J. B. Yang, K. Kamaraju, W. B. Yelon, W. J. James, Q. Cai and A. Bollero: Appl. Phys. Lett. 79 (2001) 1846-1848.

12) J. B. Yang, W. B. Yelon, W. J. James, Q. Cai, M. Kornecki, S. Roy, N. Ali and Ph l'Heritier: J. Phys.: Condens Matter 14 (2002) 6509-6519.
13) T. Onogi, K. Koyama and K. Watanabe: J. Jpn. Inst. Met. 71 (2007) 489-493.

14) S. Awaji, K. Watanabe and M. Motokawa: J. Crystal Growth 226 (2001) 83-87.

15) V. K. Pecharsky and K. A. Gschneider Jr.: Phys. Rev. Lett. 78 (1997) 4494-4497.

16) O. Tegus, E. Brück, K. H. J. Buschow and F. R. de Boer: Nature 415 (2002) $150-152$.

17) J. R. Miller, M. D. Bird, S. Bole, A. Bonito-Oliva, Y. Eyss, W. J. Kenney, T. A. Painter, H.-J. Schneider-Mumtau, L. T. Summers, S. W. Van Sciver, S. Welton, R. J. Wood, J. E. C. Williams, E. Bovrov, Y. Iwasa, M. Leupold, V. Stejskal and R. Weggel: IEEE Trans. Mang. 30 (1994) 1563-1570. 